\title{
观察性临床研究数据分析结果的 “陷阱”
}

刘岳鹏

徐州市中心医院

随着数据分析的结束, 得出了预测变量和结局 变量之间的因果关系。高兴之余, 紧张的神经还不 能放松, 还要确认自己有没有掉进了数据分析结果 的数个 “陷阱”之中。

第一个 “陷阱”, 即因果关系是偶然因素导致 的, 也就是常说的随机误差或者 I 类错误。自然现象, 比如血压, 都是围绕平均值水平在一定范围内波动, 样本量不足的情况下, 收集的变量可能会偏平均值 的一侧, 就会导致随机误差的产生。

第二个 “陷阱”, 因果关系是由偏倚导致的, 也 叫做系统误差。人为设计的意在阐明变量间因果关 系的这个研究 “系统” 会存在诸多的局限性 (研究设 计、抽样方法、测量方法等方面), 这种局限性有 可能导致错误的因果关系。

第三个 “陷阱”, 因果关系是由混杂因素导致的。 混杂因素是预测变量和结局变量之外的第三因素。 第三因素既是结局发生的原因 (在其它的研究中会被 作为预测变量), 又与预测变量存在关联 (因果关系、 共存关系等), 且预测变量不是第三因素发生的原 因。疾病的某个结局往往都是由多种因素共同导致 的, 或者说存在多个可以作为预测变量的因素, 如 果两个或多个预测因素关系较为密切, 往往同时出 现, 可能会干扰到各自对疾病结局的贡献。

以上可能的误差不可能完全避免, 比较重要 的是对存在的误差进行客观的评估和评价, 以得出 正确的结论。首先, 随机误差发生, 主要受到样本 量和测量精确度的影响, 其导致错误的因果关系的 可能性可以通过计算 $P$ 值和置信区间来评估。 $P$ 值 越小说明由偶然导致的错误的因果关系的可能性越
小, 代表有足够的样本量和足够的测量精确度, 同 样, 置信区间越窄说明由偶然导致的错误的因果关 系的可能性越小, 代表有足够的样本量和足够的测 量精确度。其次, 偏倚是无法完全避免的, 有效的 方式是在试验设计和实施过程中, 充分进行人员培 训, 保证有经验专家的全程指导。由偏倚导致的错 误的因果关系的评估则可以通过评估此研究与其它 研究, 尤其是那些使用不同研究设计的试验的一致 性来进行估计。发表的论文的 “讨论” 部分的最后 也要求专门就试验的偏倚进行讨论。最后, 混杂因素 的影响对因果关系的影响是可以通过多种方法进行 消除。需要注意的是, 多数的方法都要求对混杂因 素进行测量, 所以试验设计过程中认识到并测量可 能的混杂因素是处理混杂因素的关键。常见的措施 有: 1. 限制, 在纳入标准中对可能的混杂因素进行 排除; 2. 匹配 (常见于病例对照研究中), 选择具有 相同混杂变量值的病例和对照预防混杂产生; 3. 分 层, 按照潜在混杂变量值的水平将研究对象分隔到 不同的层; 4. 统计学调整, 采用多变量分析技术来 排除混杂变量的影响, 如多元回归、Cox 回归等; 5. 倾 向评分, 特别适用于采用观察性研究评价治疗效力 时, 控制指示性混杂。采用倾向性评分是通过建立 多变量模型来预测接受治疗的可能性, 然后每个研 究对象对得到一个预期治疗的可能性, 即 “倾向评 分”。该评分可作为分层分析或多变量分析中唯一 的混杂变量。

充分考虑到影响我们得出正确的、真实存在的 因果关系的各种因素, 并在文章报告中进行客观的 评估和阐述, 才是对试验负责的一种做法。 